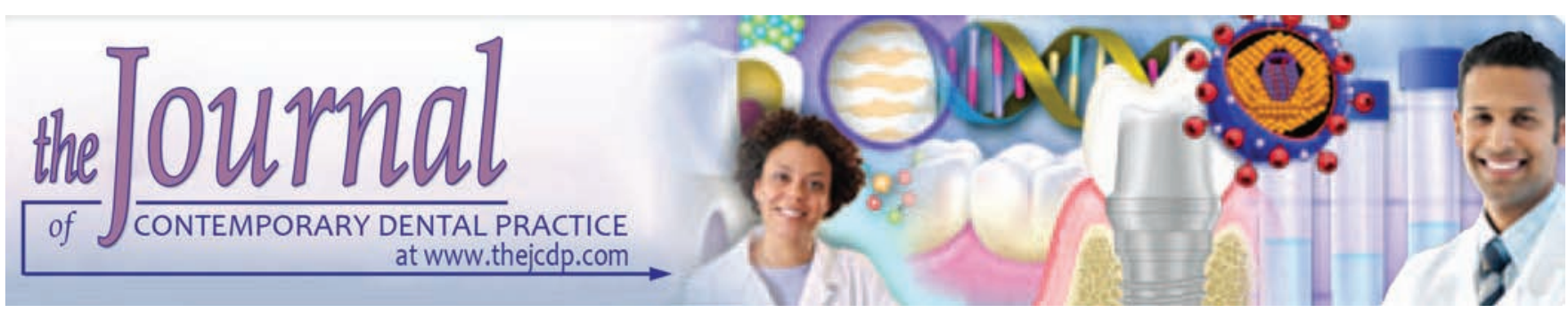

\title{
Post-insertion Posterior Single-implant Occlusion Changes at Different Intervals: A T-Scan Computerized Occlusal Analysis
}

${ }^{1}$ Azam S Madani, ${ }^{2}$ Mohammadreza Nakhaei, ${ }^{3}$ Malihe Alami, ${ }^{4}$ Hamidreza Rajati Haghi, ${ }^{5}$ Saied Mostafa Moazzami

\begin{abstract}
Aim: The aim of this prospective cohort study was to evaluate the postinsertion posterior single-implant occlusion changes at 3- and 6-month intervals using T-Scan computerized occlusal analysis.
\end{abstract}

Materials and methods: A total of 21 patients received single implant, opposed by natural dentition, in posterior regions of the maxilla or mandible (13 premolar, 8 molar) and were finally restored with cemented-retained metal-ceramic crowns. The occlusal contacts were equilibrated according to the implantprotective occlusion concept to develop light contact with heavy occlusion and no contact with light occlusion in maximum intercuspation. The percentage of force applied to the implant crowns (POFI) and contralateral teeth (POFT) was evaluated using T-Scan computerized occlusal analysis at prosthesis insertion, 3- and 6-month follow-up appointments. The data were statistically analyzed using Friedman test and Wilcoxon post hoc test $(\alpha=0.05)$.

Results: The POFI values at the 6- and 3-month follow-up appointments were significantly higher than those at prostheses insertion ( $p=0.001$ and $p=0.005$ respectively). In addition, there were significant differences between the POFI at 3- and 6 -month follow-up $(p=0.020)$. However, the POFT values at 3- and 6-month follow-up appointments were significantly lower than those at baseline $(p<0.001)$.

Conclusion: The intensity of occlusal contacts of implantsupported prostheses opposed by natural dentition gradually

\footnotetext{
1,2,4 Department of Prosthodontics, Faculty of Dentistry, Mashhad University of Medical Sciences, Mashhad, Islamic Republic of Iran

${ }^{3}$ Department of Prosthodontics, Faculty of Dentistry, Golestan University of Medical Sciences, Gorgan, Islamic Republic of Iran

${ }^{5}$ Department of Restorative Dentistry, Faculty of Dentistry Mashhad University of Medical Sciences, Mashhad, Islamic Republic of Iran

Corresponding Author: Mohammadreza Nakhaei, Department of Prosthodontics, Faculty of Dentistry, Mashhad University of Medical Sciences, Mashhad, Islamic Republic of Iran Phone: +985138829501, e-mail: Nakhaeemr@mums.ac.ir/ mrn_nakhaei@yahoo.com
}

increased after prosthesis insertion. Placement of single posterior implant-supported restoration decreased the percentage of occlusal force applied to contralateral arch.

Clinical significance: A periodic occlusal adjustment of implant-supported prostheses is necessary to prevent potential overloading from the movement of opposing natural dentition.

Keywords: Computerized occlusal analysis, Dental implant, Implant-supported prostheses, Occlusion.

How to cite this article: Madani AS, Nakhaei M, Alami M, Haghi HR, Moazzami SM. Post-insertion Posterior Singleimplant Occlusion Changes at Different Intervals: A T-Scan Computerized Occlusal Analysis. J Contemp Dent Pract 2017;18(10):927-932.

Source of support: This study was supported by a grant (900341) from the Research Council of Mashhad University of Medical Sciences. The results presented here are based on a postgraduate thesis (No. 510) submitted to Mashhad School of Dentistry and Dental Research Center.

\section{Conflict of interest: None}

\section{INTRODUCTION}

Dental implants are assumed to be more susceptible to occlusal overloading compared with natural teeth due to the absence of periodontal ligaments, which have a shock-absorbing function. ${ }^{1,2}$ Occlusal overloading is considered a potential cause of peri-implant bone loss and implant prosthesis failure ${ }^{3,4}$ and may result in mechanical complications, such as screw loosening, screw fracture, prosthesis fractures, and fracture of veneering materials. ${ }^{5,6}$ It has been demonstrated that in the presence of inflammation, overloading exacerbates plaque-induced bone resorption around implants. ${ }^{7-10}$

Dental implants also exhibit low proprioceptive feedback and low tactile sensitivity due to the absence of periodontal mechanoreceptors. ${ }^{11,12}$ Therefore, due to the weaker protective mechanism in dental implants, it 
seems that the occlusion concepts for implant-supported prostheses must be modified to reduce the occlusal forces and offer some protection.

The mean values of vertical displacement of natural teeth are 25 to $100 \mu \mathrm{m}$, while the range of axial movement of dental implants is approximately 3 to $5 \mu \mathrm{m} .{ }^{3,13}$ Due to this mobility difference between implants and teeth, the concept of implant-protective occlusion (IPO) proposes that occlusal contacts should be examined with patients in light and heavy occlusion when the natural teeth and implants are in the same jaw. No contact at heavy occlusion and light contact at light occlusion are considered to have an equal distribution of occlusal forces on teeth and implant. ${ }^{14-16}$ Although this proposition is practical in the clinical setting, the stability of the provided occlusal contacts is questionable. Implant-opposing natural teeth are likely to move, and, consequently, cause changes in the provided occlusal contacts over time, which in turn can potentially contribute to noxious forces.

Dario $^{17}$ reported that in $50 \%$ of studied patients, the occlusion of implant-supported fixed prostheses changed within 18 months after placement. Most of the changes occurred within the first 6 months after prostheses insertion. However, no further longitudinal clinical studies have been found regarding the occlusal changes in implantsupported fixed prostheses. The assessment of occlusal changes requires a method that can quantitatively measure the occlusal contacts at different intervals. None of the conventional methods, such as the use of articulation paper, shim-stock foil, and impression waxes were able to quantify occlusal contacts. Studies found no scientific correlation between the articulation paper mark size and the amount of applied force, which demonstrates the inadequacy of articulation paper in interpreting the occlusal load. ${ }^{18-20}$

First introduced in 1987, T-Scan (Tekscan, Inc., South Boston, MA, USA) is a computer-assisted dental occlusion analyzer, i.e., able to record quantifiable relative occlusal force and contact time sequencing in real time. ${ }^{18,21,22}$ A vast body of evidence supports the acceptable accuracy of computerized occlusal analysis as an occlusal indicator. ${ }^{18,21-24}$

The aim of this study was to evaluate postinsertion posterior single-implant restoration occlusion changes at different intervals using the T-Scan III computerized occlusal analysis system. The null hypothesis was that the occlusion developed at placement of single implantsupported prostheses opposed by natural teeth would not change over time.

\section{MATERIALS AND METHODS}

This prospective cohort investigation was conducted in the Department of Prosthodontics, School of Dentistry, Mashhad University of Medical Sciences, Iran. The design of this study was approved by the Ethics Committee of the Vice Chancellor for Research at Mashhad University of Medical Sciences. Informed consent was obtained from all the participants. Twenty-five males and females aged 18 to 48 years who required single-implant-supported restoration in the premolar or molar region of the posterior mandible or maxilla were recruited.

The inclusion criteria were as follows: (1) Good medical and psychological health as documented by self-assessment, (2) cusp-marginal ridge occlusal arrangement, (3) presence of all teeth in the maxilla and mandible except the one which was to be replaced with an implant, (4) absence of uncontrolled or untreated periodontal disease, and (5) acceptance of further follow-up.

The exclusion criteria were as follows: (1) Inadequate bone availability, (2) severe craniomandibular disorders, (3) use of known drugs that would affect the central nervous system or neuromuscular function, (4) severe systemic diseases or known mental disorders, (5) endodontic lesions sensitive to percussion, (6) anterior open occlusion, and (7) history of bruxism.

Implants (Biohorizons, Alabama, USA) with an internal hexagon were placed according to a standard two-stage protocol. After a healing period (at least 3 months), prosthetic treatment was accomplished and cemented-retained metal-ceramic single crowns were fabricated. The prosthetic procedure was performed by an experienced prosthodontist. Impressions were taken with a polyvinyl siloxane (Panasil; Kettenbach Dental, Eschenburg, Germany) using stock trays and one-step putty/wash pick-up technique. Implant analogs were attached and the impressions were poured with type IV die stone (Herostone, Vigodent Inc., Rio de Janeiro, Brazil) according to the manufacturer's instructions. When set (120 minutes after pouring), the impressions were separated from the casts. The prefabricated titanium abutments were attached to the analogs and casts were scanned using a three-dimensional (3D) laser scanner (TRIOS, 3 Shape, Copenhagen, Denmark). The 3D designs of frameworks were developed with software. All frameworks were milled from presintered hard Co-Cr blocks (CORITEC Co-Cr disk, imes-icore $\mathrm{GmbH}$, Germany) using a 5-axis milling machine (CORITEC 450i, imes-icore GmbH Im Leibolzgraben, Germany). The frameworks were checked intraorally for the accuracy of fit. The margins were inspected with a dental explorer and the internal fit was evaluated with a silicone disclosing agent (Fit Checker; GC America Inc, IL, USA). After a satisfying try-in, the occlusal surfaces of the frameworks were veneered with layering porcelain. During the porcelain try-in appointment, the proximal contacts were evaluated with waxed dental floss (Essentialfloss, Oral-B, Ireland) and articulation foil (Gnatho-Film, Dr Jean Bausch GmbH \& Co., Köln, Germany). 
Occlusal contact was equilibrated in static and dynamic movement according to IPO concept. A 12 um shim stock (PROGRESS, Dr Jean Bausch GmbH \& Co., Köln, Germany) was held intraorally using Miller forceps (Hu-Friedy Mfg. Co., Chicago, Illinois, USA), and the patient was asked to occlude into the strip in maximum intercuspation position. Ability to hold the strip between opposing teeth indicated the occlusal contacts. The implant crown was then inserted again, and it was assessed whether the patients could still hold the strip between opposing teeth. A $100 \mu \mathrm{m}$ articulating paper (PROGRESS, Dr Jean Bausch GmbH \& Co., Köln, Germany) was coated with a thin layer of petroleum jelly and used to identify the location of occlusal interferences on the implant crown. Any premature contact was eliminated using fine and extra-fine diamond burs (Brasseler Komet, Lemgo, Germany). Then, a $16 \mu \mathrm{m}$ articulating paper (Gnatho-Film, Dr Jean Bausch GmbH \& Co., Köln, Germany) was used for initial implant occlusal adjustment in occlusion under light biting force. The patient was asked to lightly tap his/her teeth together through the articulation strip five times in succession. The occlusal contact was equilibrated so that the implant prosthesis would barely contact the opposing tooth, while the adjacent teeth in the arch exhibited greater occlusal contacts.

After equilibration with light bite force, the patient was asked to tap his/her teeth through the articulating paper with maximum occlusal force. The purpose of occlusion evaluation under heavy occlusal load was to ensure the contact points showed similar intensity on the implant crown and adjacent teeth. In protrusive and lateral movements, the excursive contacts on the implant prosthesis were also eliminated.

At the insertion appointment, the implant abutment screws were tightened with a torque of $35 \mathrm{Ncm}$, according to the implant manufacturer's guidelines. The restorations were intraorally validated regarding the marginal fit and proximal contact. The occlusion was controlled in both centric and lateral excursions and adjusted if necessary. Finally, all the implant crowns were cemented using temporary cement (Temp Bond, Kerr, Salerno, Italy). In case of necessary readjustments, the restoration was sent back to the laboratory for glazing and cemented on the same day.

\section{T-Scan III Bite Recording Procedure}

The occlusion was assessed using a T-Scan III computerized occlusal analysis system (Tekscan, Inc., South Boston, MA, USA) after cementation of implant crowns (baseline). This device uses a U-shaped pressure-sensitive sensor, i.e., placed intraorally between dental arches. When a patient occludes on the sensor, occlusal data are captured, processed by the related software, and the outputs are displayed as two-dimensional and 3D illustrations. The percentages of applied forces per tooth are represented as bars and columns on the same tooth in graphical displays. The width of the maxillary central incisor in each patient was measured using a digital caliper to accurately simulate the dimensions of each subject's dental arch.

According to the manufacturer's recommendation, before recording any occlusal force data, the sensitivity level of the T-Scan was adjusted to match the range of occlusal force in each individual. The correct sensitivity was selected by limiting the number of red/pink high-force columns/contacts observed on the graphical display, to a maximum of three during prerecording test conditions. The patients were then asked to occlude the recording sensor with the maximum possible bite force for 1 to 2 seconds. This procedure was repeated three times; the closure with maximum occlusal force was selected for analysis. The percentage of applied occlusal forces displayed on the implant crown and contralateral tooth was recorded.

Three and 6 months after the initial visit, occlusion in each patient was again assessed using T-Scan III according to the previously mentioned procedure (Fig. 1). The percentage of applied occlusal force to the implant (POFI) crowns and contralateral teeth (POFT) was statistically analyzed using the Friedman test. Wilcoxon test $(\alpha=0.05)$ was used to compare the POFI or POFT values when a significant difference was observed.

\section{RESULTS}

Of 25 participants, 3 were excluded due to lack of cooperation in posttreatment follow-ups, and 1 was excluded due to restorative treatments and the subsequent changes in occlusion within the 6-month study period. In the remaining 21 patients (10 females and 11 males), the implant crown was located in the first premolar $(n=13)$ or molar region $(n=8)$. The mean \pm standard deviation (SD) age of patients at prosthesis insertion was $30.81 \pm 8.85$ years (age range $=18-48)($ Table 1$)$.

The mean values and SDs of POFI and POFT are presented in Table 2. The Friedman test revealed significant differences among the POFI values obtained 0,3 , and 6 months after prosthesis placement $(\mathrm{p}<0.001)$. According to Wilcoxon test, POFI values at 6- and 3-month followup appointments were significantly higher than those at prostheses insertion ( $\mathrm{p}=0.001$ and $\mathrm{p}=0.005$ respectively). Significant differences were also noted between the POFI values obtained at 3 and 6 months after prostheses placement $(p=0.020)$.

The Friedman test demonstrated significant differences among the POFT values recorded 0,3 , and 6 months after prosthesis insertion $(\mathrm{p}<0.001)$. Based on the Wilcoxon test, the POFT values at 3- and 6-month 


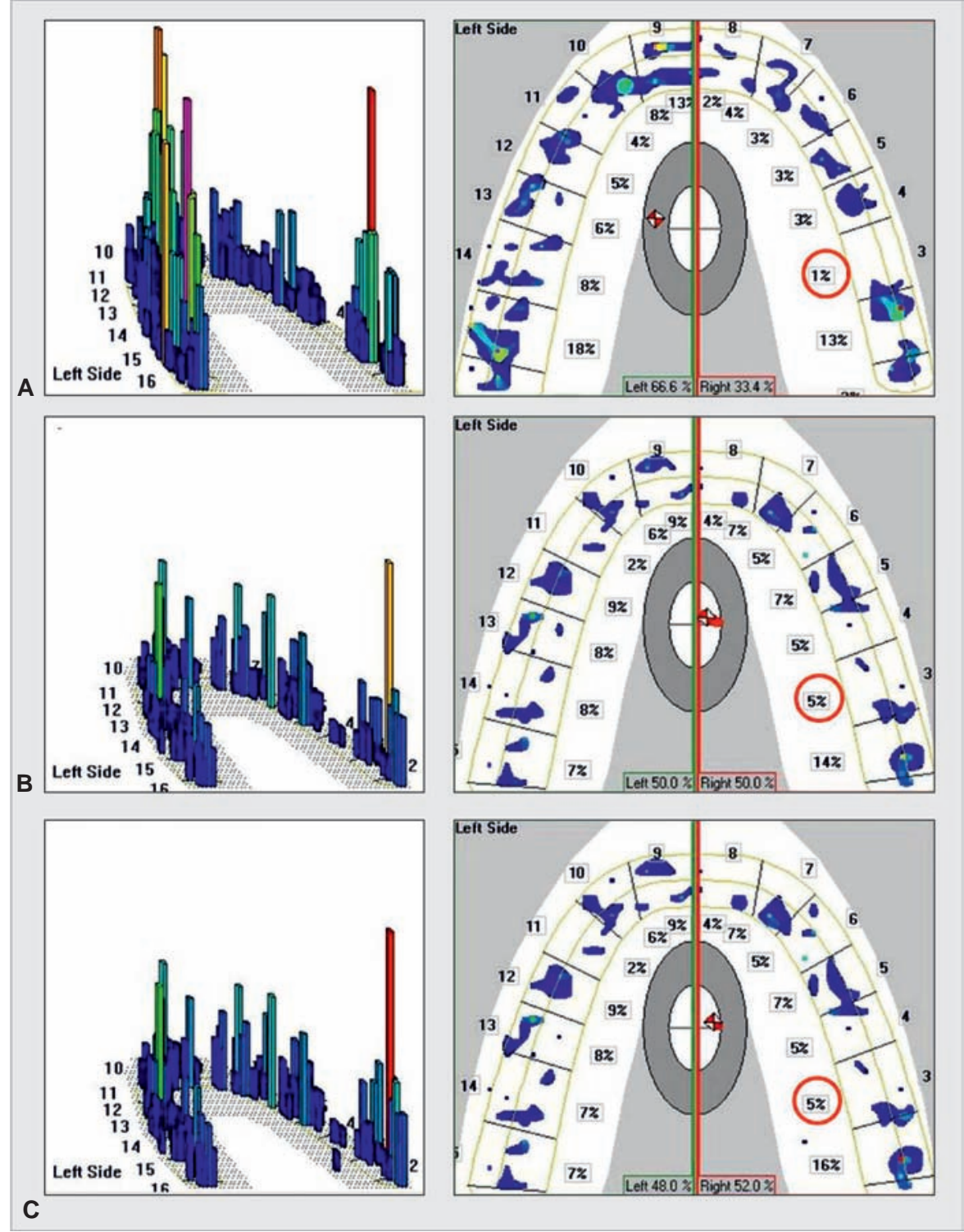

Figs 1A to C: T-Scan occlusal analysis of a participant at three different intervals. Note that tooth no. 3 (maxillary right first molar) was replaced by an implant-supported crown. (A) Prosthetic placement appointment (baseline measurement); (B) three-month follow-up; and (C) six-month follow-up

Table 1: Demographic data

\begin{tabular}{|c|c|c|c|c|c|c|c|c|}
\hline \multicolumn{2}{|c|}{ Gender } & \multicolumn{2}{|c|}{ Jaw } & \multicolumn{2}{|c|}{ Location } & \multicolumn{3}{|c|}{ Age } \\
\hline Female & Male & Maxilla & Mandible & Premolar & Molar & Mean & Minimum & Maximum \\
\hline 10 & 11 & 9 & 12 & 13 & 8 & 30.81 & 18 & 50 \\
\hline
\end{tabular}

Table 2: Mean (SD) values for POFI and POFT at different intervals

\begin{tabular}{llll}
\hline & $\begin{array}{l}\text { Prosthetic insertion } \\
\text { (baseline) }\end{array}$ & $\begin{array}{l}\text { 3-Month } \\
\text { follow-up }\end{array}$ & $\begin{array}{l}\text { 6-Month } \\
\text { follow-up }\end{array}$ \\
\hline POFI & $4(0.19)^{\mathrm{a}}$ & $4.52(0.20)^{\mathrm{b}}$ & $5(0.28)^{\mathrm{c}}$ \\
POFT & $9.47(0.28)^{\mathrm{a}}$ & $8.71(0.35)^{\mathrm{b}}$ & $8.23(0.30)^{\mathrm{b}}$ \\
\hline
\end{tabular}

Different uppercase letters in the row mean that the values are significantly different $(p<0.05)$

follow-up appointments were significantly lower than those at baseline $(p<0.001)$. However, there were no significant differences between the POFT values at 3 - and 6-month follow-up ( $\mathrm{p}=0.061$ ).

\section{DISCUSSION}

According to the results of this study, the intensity of occlusal loads applied to the implant-supported prostheses opposed by natural teeth increased gradually over time. Thus, the null hypothesis, that the occlusion developed at the placement of implant-supported prostheses would not change, is rejected.

These findings confirm the theory that teeth keep erupting as they face a resistance equal to their eruptive force. ${ }^{25}$ The implant-opposing natural dentition continues to erupt so that appropriate contact with the 


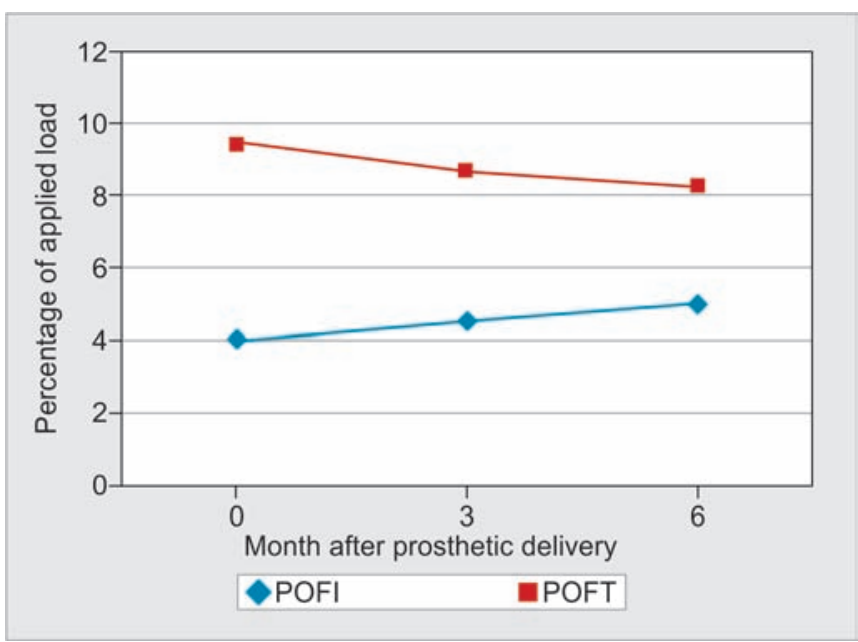

Graph 1: Changes in the percentage of force applied to the implant crowns and contralateral teeth at 3-month intervals

implant-supported prosthesis is established. It can create contacts similar to those of the natural teeth.

The findings of this study are consistent with those observed by Dario, ${ }^{17}$ who used T-Scan to assess the changes in occlusion of implant-supported fixed prostheses at 3-month intervals after prosthesis placement. It was reported that in $46 \%$ of patients, the occlusal load applied to implant prostheses changed within 18 months after prosthesis placement, and almost one-third of these changes took place during the first 6 months.

Although the results of this study showed that the force applied to the implant restoration increased gradually, the increase was higher in the first 3 months than in the subsequent 3 months. However, the question remains as to when this gradual increase in applied force will come to an end.

In the present study, increases in mean POFI values were accompanied by a decrease in mean POFT values in the contralateral arch (Graph 1). This finding is consistent with the result of Roque et al, ${ }^{26}$ who demonstrated that placement of single posterior implant-supported restorations opposed by natural dentition decreased the percentage of total occlusal pressure in the contralateral arch. This indicates the unity of the whole dental arch to distribute each force, as well as the influence of each tooth on the intensity of the applied force on the other teeth in the same jaw.

The IPO concept recommends light contact at heavy closure and no contact at light closure when there are implants and natural teeth in the same jaw. This concept was originally formulated based on the differences between the amount of natural teeth and implant movement. ${ }^{12,15,16}$ Nevertheless, there is neither a randomized controlled trial nor a prospective study directly comparing the IPO concept and occlusion concepts recommended for natural dentition. ${ }^{1}$ It is not even clear how much deviation from the IPO criteria can be tolerated by implant-supported prostheses.

Animal studies have shown conflicting results regarding the influence of excessive occlusal load on osseointegrated dental implants. It is known that occlusal overload may lead to marginal bone loss in the presence of periimplant tissue inflammation. Nonetheless, it is unclear whether occlusal overload causes marginal bone loss in the absence of inflammation. Even in the absence of periimplant tissue inflammation, an increase in bone density was reported around the implants subjected to occlusal overload. The intensity of overload is also crucial and should be in the biologically acceptable range. However, this biological range is also unknown. ${ }^{8-10,27}$ In an animal study, overloading was mimicked by the excessive height of the prostheses. The results showed that in an uninflamed peri-implant environment, the threshold of excessive height of the superstructures at which bone resorption may initiate around the implants, is approximately $180 \mu .{ }^{4}$ Wennerberg et $\mathrm{al}^{28}$ reported a considerable divergence of the "optimal occlusion" in patients with mandibular implant-supported fixed prostheses opposed by complete dentures, which had no negative consequence on the clinical and radiographically recorded variables.

It should be noted that other variables also affect the long-term occlusion stability of implant-supported prostheses. Continuous eruption of adjacent natural teeth throughout life could result in infraocclusion of dental implants in the long term. An additional effective variable is tooth wear. Natural dentition wear affects natural enamel and dentin at a far greater rate than the prosthesis veneering materials which can cause premature adverse occlusion in implant-supported prostheses. ${ }^{29-31}$

This study is one of the few which assessed occlusion changes in implant-supported prostheses in a 6-month period. However, due to lack of patient cooperation, the study could not be continued for a longer period. One of the advantages of this study was the assessment of changes in a subject to eliminate interfering variables and the use of T-Scan for quantitative evaluation of these changes. One of the challenges in using T-Scan is that it is time consuming and requires a skilled operator. One of the limitations of T-Scan is that despite providing the percentage of force, it does not have the capacity to measure the absolute bite force.

The current study investigated one of the suggested guidelines for implants in the short term. Similar to previous studies, the results showed the necessity of periodic occlusal adjustments to prevent potential overloading from the positional changes of teeth. ${ }^{1,3,4}$ There is insufficient evidence to establish clinical guidelines for implant occlusion. Thus, randomized clinical trials are needed to assess the effect of occlusion changes on the implant surrounding marginal bone and the implant components. 


\section{CONCLUSION}

Within the limitations of this study, the following conclusions were drawn:

- The intensity of occlusal contacts of implantsupported prostheses opposed by natural dentition gradually increased after prosthesis insertion

- Placement of single posterior implant-supported restorations decreased the percentage of applied occlusal force to the contralateral arch.

\section{CLINICAL SIGNIFICANCE}

A periodic occlusal adjustment of implant-supported prostheses is necessary to prevent potential overloading from the movement of opposing natural dentition.

\section{REFERENCES}

1. Koyano K, Esaki D. Occlusion on oral implants: current clinical guidelines. J Oral Rehabil 2015 Feb;42(2):153-161.

2. Yuan JC, Sukotjo C. Occlusion for implant-supported fixed dental prostheses in partially edentulous patients: a literature review and current concepts. J Periodontal Implant Sci 2013 Apr;43(2):51-57.

3. Kim Y, Oh TJ, Misch CE, Wang HL. Occlusal considerations in implant therapy: clinical guidelines with biomech ration Clin Oral Implants Res 2005 Feb;16(1):26-35.

4. Miyata T, Kobayashi Y, Araki H, Ohto T, Shin K. The influence of controlled occlusal overload on peri-implant tissue. Part 3: a histologic study in monkeys. Int J Oral Maxillofac Implants 2000 May-Jun;15(3):425-431.

5. Zarb GA, Schmitt A. The longitudinal clinical effectiveness of osseointegrated dental implants: the Toronto study. Part II: the prosthetic results. J Prosthet Dent 1990 Jul;64(1):53-61.

6. Schwarz MS. Mechanical complications of dental implants. Clin Oral Implants Res 2000;11(Suppl 1):156-158.

7. Kozlovsky A, Tal H, Laufer BZ, Leshem R, Rohrer MD, Weinreb M, Artzi Z. Impact of implant overloading on the peri-implant bone in inflamed and non-inflamed peri-implant mucosa. Clin Oral Implants Res 2007 Oct;18(5):601-610.

8. Duyck J, Vandamme K. The effect of loading on peri-implant bone: a critical review of the literature. J Oral Rehabil 2014 Oct;41(10):783-794.

9. Chambrone L, Chambrone LA, Lima LA. Effects of occlusal overload on peri-implant tissue health: a systematic review of animal-model studies. J Periodontol 2010 Oct;81(10): 1367-1378.

10. Naert I, Duyck J, Vandamme K. Occlusal overload and bone/ implant loss. Clin Oral Implants Res 2012 Oct;23 (Suppl 6): 95-107.

11. Kazemi M, Geramipanah F, Negahdari R, Rakhshan V. Active tactile sensibility of single-tooth implants versus natural dentition: a split-mouth double-blind randomized clinical trial. Clin Implant Dent Relat Res 2014 Dec;16(6):947-955.

12. Schulte W. Implants and the periodontium. Int Dent J 1995 Feb;45(1):16-26.

13. Gross MD. Occlusion in implant dentistry. A review of the literature of prosthetic determinants and current concepts. Aust Dent J 2008 Jun;53 (Suppl 1):S60-S68.
14. Lundgren D, Laurell L. Biomechanical aspects of fixed bridgework supported by natural teeth and endosseous implants. Periodontol 20001994 Feb;4:23-40.

15. Carlsson GE. Dental occlusion: modern concepts and their application in implant prosthodontics. Odontology 2009 Jan;97(1):8-17.

16. Misch, CE.; editor. Occlusal consideration for implantsupported prostheses: implant protective occlusion. In: Dental implant prosthetics. 2nd ed. St Louis (MO): Mosby Elsevier; 2015. p. 874-912.

17. Dario LJ. How occlusal forces change in implant patients: a clinical research report. J Am Dent Assoc 1995 Aug;126(8): 1130-1133.

18. Qadeer S, Kerstein R, Kim RJ, Huh JB, Shin SW. Relationship between articulation paper mark size and percentage of force measured with computerized occlusal analysis. J Adv Prosthodont 2012 Feb;4(1):7-12.

19. Carey JP, Craig M, Kerstein RB, Radke J. Determining a relationship between applied occlusal load and articulating paper mark area. Open Dent J 2007 Jul;1:1-7.

20. Saad MN, Weiner G, Ehrenberg D, Weiner S. Effects of load and indicator type upon occlusal contact markings. J Biomed Mater Res B Appl Biomater 2008 Apr;85(1):18-22.

21. Afrashtehfar KI, Qadeer S. Computerized occlusal analysis as an alternative occlusal indicator. Cranio 2016 Jan;34(1):52-57.

22. Qadeer S, Yang L, Sarinnaphakorn L, Kerstein RB. Comparison of closure occlusal force parameters in post-orthodontic and non-orthodontic subjects using T-Scan ${ }^{\circledR}$ III DMD occlusal analysis. Cranio 2016 Nov;34(6):395-401.

23. Koos B, Godt A, Schille C, Göz G. Precision of an instrumentation-based method of analyzing occlusion and its resulting distribution of forces in the dental arch. J Orofac Orthop 2010 Nov;71(6):403-410.

24. Cohen-Levy J, Cohen N. Computerized analysis of occlusal contacts after lingual orthodontic treatment in adults. Int Orthod 2011 Dec;9(4):410-431.

25. Dawson, PE.; editor. Vertical dimension. In: Functional occlusion: from TMJ to smile design. 1st ed. St Louis (MO): Mosby Elsevier; 2007. p. 113-129.

26. Roque MA, Gallucci GO, Lee SJ. Occlusal pressure redistribution with single implant restorations. J Prosthodont 2016 Oct;26(4):275-279.

27. Chang M, Chronopoulos V, Mattheos N. Impact of excessive occlusal load on successfully-osseointegrated dental implants: a literature review. J Investig Clin Dent 2013 Aug;4(3):142-150.

28. Wennerberg A, Carlsson GE, Jemt T. Influence of occlusal factors on treatment outcome: a study of 109 consecutive patients with mandibular implant-supported fixed prostheses opposing maxillary complete dentures. Int J Prosthodont 2001 Nov-Dec;14(6):550-555.

29. Heij DG, Opdebeeck H, van Steenberghe D, Kokich VG, Belser U, Quirynen M. Facial development, continuous tooth eruption, and mesial drift as compromising factors for implant placement. Int J Oral Maxillofac Implants 2006 Nov-Dec;21(6):867-878.

30. Oesterle LJ, Cronin RJ Jr. Adult growth, aging, and the single-tooth implant. Int J Oral Maxillofac Implants 2000 Mar-Apr;15(2):252-260.

31. Daftary F, Mahallati R, Bahat O, Sullivan RM. Lifelong craniofacial growth and the implications for osseointegrated implants. Int J Oral Maxillofac Implants 2013 Jan-Feb;28(1):163-169. 